\section{Aromatic Ring Opening in the Presence of Oxygen in Irradiated Solutions}

WHEN aqueous solutions of benzene are irradiated in vacuo with $\alpha$-rays or neutrons which produce oxygen, a dialdehyde, identified as mucondialdehyde, is produced ${ }^{1}$. The same product was found also when solutions of benzene containing oxygen were irradiated with X-rays ${ }^{2}$. Thus ring opening appeared to be connected with the presence of oxygen, rather than with high radical concentrations and multiple reactions on the same benzene ring. The identification of the product as mucondialdehyde was confirmed ${ }^{3}$. Material balance cannot be obtained ${ }^{4}$ unless the formation of oxidation products other than phenol is assumed. The interesting possibility arises that aromatic ring opening in the presence of oxygen may occur as the result of a single primary radical reaction step and that mucondialdehyde is a primary product, being formed in direct competition with the formation of phenol. Analytical methods were used which enabled us to determine phenol, catechol and quinol ${ }^{1,2}$ in the presence of hydrogen peroxide ${ }^{5}$ and of mucondialdehyde, $o$. and $p$-quinone. These last three were determined after condensation with $p-\mathrm{NO}_{2}$-phenylhydrazine in $2 N$ sulphuric acid, extraction into carbon tetrachloride, re-extraction into $2 \mathrm{~N}$ sodium hydroxide and spectrophotometric measurement in $0.8 \mathrm{~N}$ sodium hydrate at $390 \mathrm{~m} \mu$ (mucondialdehyde); $510 \mathrm{~m} \mu$ (max.) and $400 \mathrm{~m} \mu$ (min.) (o-quinone); and 475 $\mathrm{m} \mu$ ( $p$-quinone). Details of the analytical methods developed will be published separately.

We have found that mucondialdehyde is indeed formed simultaneously with phenol, directly from benzene, without the previous formation of phenol, catechol, quinol or quinones. In Fig. 1 it is shown that in aqueous benzene solution irradiated with $\mathrm{X}$-rays at $p \mathrm{H}=7 \cdot 1$, while phenol is formed with an initial yield of $G=2 \cdot 69$, mucondialdehyde is being formed with an initial yield of $G=0.8$. Under the same conditions quinones are not formed in measur. able quantities. The addition of phenol to the solution before irradiation does nut increase the yield of mucondialdehyde. The ratio of $G$ (phenol) $/ G$ (mucondialdehyde) remains constant at $\sim 3$ in the range $p \mathrm{H}=0 \cdot 4-7 \cdot 5$. The absolute yield increases slightly at low $p \mathrm{H}$ values.

One possible explanation of the direct aromatic ring opening is that due to Weiss ${ }^{6}$, who assumes that the addition to benzene of one radical formed from water by the radiation leads to further addition of oxygen and formation of a hydroperoxide which, losing water, yields mucondialdehyde directly through ring opening Alternative possibilities include the interaction of an excited benzene molecule with oxygen leading to direct ring opening and dialdehyde formation.

We have therefore investigated the photochemistry of aqueous benzene solutions containing oxygen, irradiated with ultra-violet light of wave-length above 2100 A. Formation of phenol and mucondialdehyde was observed, mucondialdehyde being once again a primary product formed in competition with the formation of phenol. The ratio of phenol/mucondialdehyde however changes with changing $p \mathrm{H}$, the relative yield of mucondialdehyde decreasing strongly with decreasing $p \mathrm{H}$. The different degrees of depen. dence on $p \mathrm{H}$ would indicate that the mechanisms involved in photochemistry and radiation chemistry are not wholly identical.

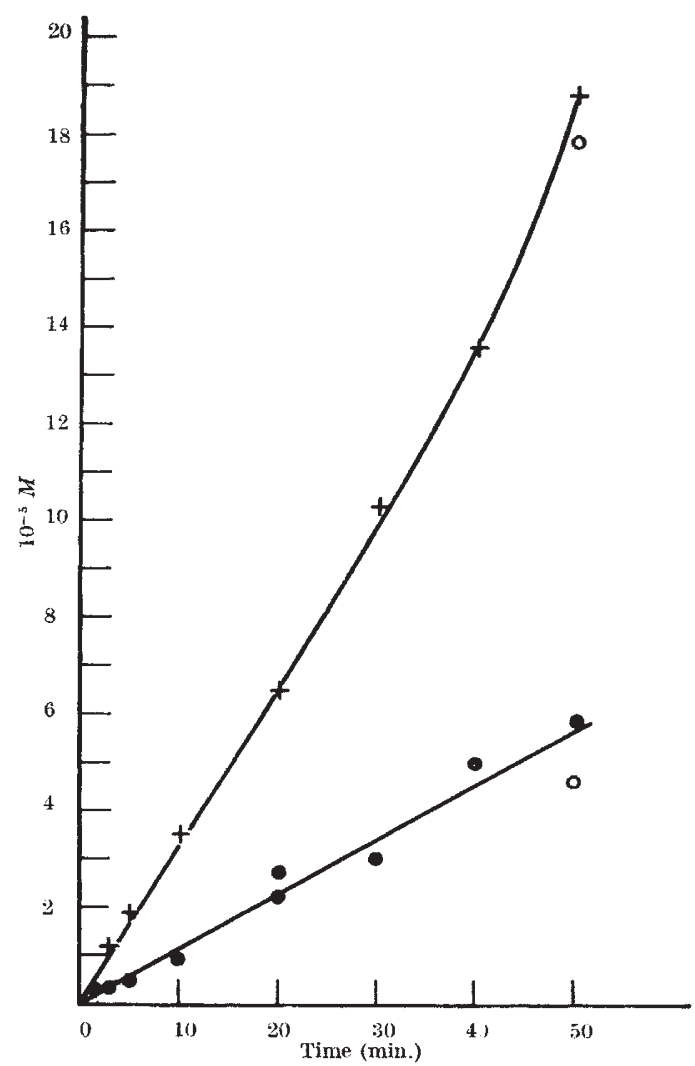

Fig. 1. Formation of phenol $(+)$ and mucondialdehyde $(O)$ in oxygenated benzene solution at $p \mathrm{H}=7 \cdot 1$. 0 , results in presenc of initially added pheno $\left(2 \times 10^{-4} M\right), 200-\mathrm{kVp}$. X-rays, $1380 \mathrm{r}$. $/ \mathrm{min}$.

It appears therefore that the interaction with molecular oxygen of both an excited benzene molecule or of a radical formed from benzene may lead in aqueous solution directly to aromatic ring opening. We are investigating the possible connexion with the known effect of oxygen on the spectrum of benzene?

Full details will be published separately.

\section{ISRAEL LOEFF} Gabriel Stein

Department of Physical Chemistry, Hebrew University, Jerusalem.

Stein, G., and Weiss, J., J. Chem. Soc., 3245 (1949).

Stein, G,, and Weiss, J., J. Chem. Soc,, 3265 (1951)

Daniels, M., Scholes, G., and Weiss, J., J. Chem. Soc., 832 (1956).

Baxendale, J. H., and Smithies, D., J. Chem. Soc., 779 (1959).

Lochanadel, C. J., J. Phys. Chem., 56, 587 (1952). Eisenberg, G, M. Ind. Eng. Chem., 15, 327 (1943).

Weiss, J., "Actions Chimiques des Radiation", 4, 42 (Masson et cie.

7 Evans, 1). F,J. Chem. Suc., 1351, 3885 (1957).

\section{BIOCHEMISTRY}

\section{Isolation of 2-Aminoethane Phosphonic Acid from Rumen Protozoa}

Is the course of experiments on the amino-acid composition of rumen Protozoa, an unknown ninhy. drin-positive substance was found by paper chromatography to be present in acid hydrolysates of the ether-ethanol soluble fraction of Protozoa. The substance was isolated in crystalline form and identified as 2-amino-ethane phosphonic acid, 